\title{
Evaluation of right ventricular functions in patients with acute pulmonary embolism
}

We read with great interest the article by Gromadziński et al. [1] entitled "The influence of acute pulmonary embolism on early and delayed prognosis for patients with chronic heart failure", which published in Cardiology Journal. They aimed to evaluate the potential impact of acute pulmonary embolism (APE) on early and long-term prognosis in patients with chronic heart failure (CHF). In this study, right ventricle (RV) dysfunction was assessed by right ventricular end-diastolic dimension (RVEDD), maximal tricuspid regurgitation pressure gradient (TRPG) and tricuspid annular plane systolic excursion (TAPSE) in a two-dimensional (2D) echocardiography. They confirmed APE were characterized by higher RVEDD and decreased RV contractility assessed with TAPSE, and the patients in both groups did not differ in TRPG value. Finally, they showed recent episode of pulmonary embolism in patients with $\mathrm{CHF}$ is an independent risk factor for early mortality in a 6-month follow-up.

Assessing RV morphology and function is of paramount importance in diseases such as pulmonary embolism, pulmonary hypertension and myocardial infarction involving the RV. In patients with APE, RV dysfunction on the echocardiogram is an independent and powerful predictor of early death in patients with APE [2].

Echocardiography, being non-invasive, widely available, relatively inexpensive, and having no side effects, is the modality of choice for the assessment of morphology and function of the RV in clinical practice. Echocardiographic volume and function assessment of the RV is complicated by the complex geometry of this chamber, the pronounced trabeculation that compromises accurate endocardial delineation, and the anterior position that often limits echo image quality [3].

Unlike the left ventricle, where biplane methods are accepted and widely used for a global assessment of systolic function, identification of functional abnormalities on the basis of visual echocardiographic assessment solely is inaccurate, frequently resulting in false-positive findings [4].
Owing to the incomplete visualization and evaluation of the RV, in addition to RVEDD, TAPSE and TRPG values, combined projections such as $3 \mathrm{D}$ echocardiography, right ventricular fractional area change, myocardial performance index (MPI, Tei index), RV dP/dt, tissue Doppler myocardial imaging techniques, strain rates, acceleration time of pulmonary artery, hepatic flow patterns, and inferior vena caval diameters are needed for a comprehensive evaluation of RV structure and function.

And also, release of troponin can occur in patients with pulmonary embolism in the absence of angiographic coronary artery disease due to an abrupt increase in RV wall tension with compression of the right coronary artery and direct myocardial micro-injury. Levels of cardiac biomarkers can be used to predict RV dysfunction and clinical outcome [5].

In conclusion, RV dysfunction is key and has prognostic value for risk stratification in APE. If combinations of RV echocardiographic parameters and cardiac biomarker levels in evaluating RV function were used and information of baseline hemodynamic parameters and extent of perfusion defects were given; the study could have been more valuable and predicted clinical outcomes more accurately.

\section{Conflict of interest: none declared}

\section{References}

1. Gromadziński L, Targoński R, Januszko-Giergielewicz B, Ciurzyński M, Pruszczyk P. The influence of acute pulmonary embolism on early and delayed prognosis for patients with chronic heart failure. Cardiol J, 2012; 19: 625-631.

2. Braude S, Martens-Nielsen J. Severe refractory hypoxaemia in submassive pulmonary embolism: A surrogate marker of severeright ventricular dysfunction and indication for thrombolysis. Intern Med J, 2012; 42: 712-715.

3. D’Oronzio U, Senn O, Biaggi P et al. Right heart assessment by echocardiography: Gender and body size matters. J Am Soc Echocardiogr, 2012; 25: 1251-1258.

4. Jurcut R, Giusca S, La Gerche A, Vasile S, Ginghina C, Voigt JU. The echocardiographic assessment of the right ventricle: What to do in 2010? Eur J Echocardiogr, 2010; 11: 81-96.

5. Henzler T, Roeger S, Meyer M et al. Pulmonary embolism: CT signs and cardiac biomarkers for predicting right ventricular dysfunction. Eur Respir J, 2012; 39: 919-926.

\section{Emre Yalcinkaya ${ }^{1}$, Murat Celik ${ }^{1}$,Baris Bugan ${ }^{2}$, Uygar Cagdas Yuksel ${ }^{1}$ \\ ${ }^{1}$ Gulhane Military Medical Faculty, Department of Cardiology, GATA Etlik 06018 Ankara, Turkey, tel: +905336577191 (Mobile), +903123044257 (Work), fax: +903123044250,e-mail: dremreyalcinkaya@gmail.com

